\title{
Synthesis and Characterization of Gold Nanoparticles-Antibody- Enzyme Conjugate for use in Influenza a Spesific Nano-ELISA
}

\author{
Yasemin Budama-Kilinc, Rabia Cakir-Koc, Guncem Ozgun Eren \\ Bioengineering Department, Yildiz Technical University \\ A-122 Davutpasa Campus, 34220, Istanbul, Turkey \\ yaseminbudama@gmail.com; budama@yildiz.edu.tr; rabiakoc@yildiz.edu.tr; guncemozgun@gmail.com
}

\section{Extended Abstract}

Influenza is the one of the most common infectious diseases cause respiratory infections. It is classified into three main categories; A, B, and C. Influenza A virus mutates frequently. It affects all age groups worldwide, and causes significant loss of workdays, human suffering, and mortality every year [1,2].

Despite the genetic differentiation of the influenza A virus, it has been shown that the peptide region formed by the 24 amino acids of the M2e protein located on the surface of the virus is the most conserved region in all strains [3-5]. Although M2e is normally found in small quantities on virus particles, it is secreted abundantly on the cell surface infected by the virus [6].

Immunoglobulin Y (IgY) is obtained from the eggs of chicken. It is the most abundant antibody that found chicken than other immunoglobulins. With the non-invasive, non-allergic, and low cost-effective process, IgY antibody is obtained more advantageous than the other antibody production metods [8].

There are different methods for detecting the Influenza A virus. Gold nanoparticles based ELISA (Nano-ELISA) is the one of important current methods [9, 10]. As gold nanoparticles play an important role in increasing signal strength, NanoELISA method is more sensitive than the traditional ELISA where a more confident results are required [10].

In this study in order to use for Influenza A diagnosis, M2e peptide spesific IgY antibodies were produced, purified with Polyethylene Glycol (PEG-6000) precipitation procedure [8] and afinity chromatography, and characterized. On the other hand, colloidal gold nanoparticles (AuNPs) were synthesized with Turkevich Method [9], and then the conjugation of AuNPs-IgY was performed and characterization of AuNPs-IgY conjugate was done with spectroscopic methods such as UV-Vis, Zeta-Sizer, FTIR and with TEM analysis. Finally, gold nanoparticles-antibody-enzyme (AuNPs-IgY-ALP) conjugate was synthesized in presence glutaraldehyde for use in Influenza A spesific Nano-ELISA.

\section{Acknowledgment}

The authors would like to thank TUBITAK for their support the named "Development of Rapid Diagnostic Kit with Immunochromotographic Method of Depending on IgY Antibody Specific in (M2e) Peptide for Diagnosis of Influenza A Infection" (Project No. 115S132).

\section{References}

[1] Centers for Disease Control and Prevention, Seasonal Influenza: Flu Basics, [Online]. Available: at http://www.cdc.gov/flu/about/disease.htm

[2] Influenza (Seasonal), World Health Organization, [Online], Available: http://www.who.int/mediacentre/factsheets/fs211/en/

[3] S. M. Tompkins, et al, "Matrix protein 2 vaccination and protection against influenza viruses, including subtype H5N1," Matrix, 2007.

[4] S. Neirynck, et al, "A universal influenza A vaccine based on the extracellular domain of the M2 protein," Nature Medicine, vol. 5, no. 10, pp. 1157-1163, 1999.

[5] M. De Filette, et al., "Improved design and intranasal delivery of an M2e-based human influenza A vaccine," Vaccine, vol. 24, no. 44, pp. 6597-6601, 2006. 
[6] S. Khurana, et al, "H5N1 SeroDetect EIA and rapid test: a novel differential diagnostic assay for serodiagnosis of H5N1 infections and surveillance," Journal of Virology, vol. 85, no. 23, pp. 12455-12463, 2011.

[7] D. Pauly, P. A. Chacana, E. G. Calzado, B. Brembs, R. Schade, "IgY technology: extraction of chicken antibodies from egg yolk by polyethylene glycol (PEG) precipitation,” JoVE (J Vis Exp), vol. 51, pp. e3084-e3084, 2011.

[8] V. D. Pham, H. Hoang, T. H. Phan, U. Conrad, H. H. Chu, "Production of antibody labeled gold nanoparticles for influenza virus H5N1 diagnosis kit development," Adv. Nat. Sci.: Nanosci Nanotechnol, vol. 3, pp. 45017-45024, 2012.

[9] Y. C. Yeh, B. Creran, V. M. Rotello, "Gold nanoparticles: preparation, properties, and applications in bionanotechnology," Nanoscale, vol. 4, no. 6, pp. 1871-1880, 2012.

[10] G. S. I mani, R. Madani, F. Golchinfar, et al, "Development of a Nano-ELISA system for the rapid and sensitive detection of H9N2 avian influenza," Archives of Razi Institute, vol. 71, pp. 29-34, 2016. 